\title{
Fine-Scale Genetic Structure and Reproductive Biology of the Blueberry Pathogen Monilinia vaccinii-corymbosi
}

\author{
Kathleen M. Burchhardt, Megan E. Miller, William O. Cline, and Marc A. Cubeta
}

Department of Plant Pathology, North Carolina State University, Raleigh, NC 27695.

Accepted for publication 15 October 2016.

\begin{abstract}
The fungus Monilinia vaccinii-corymbosi, a pathogen of Vaccinium spp., requires asexual and sexual spore production to complete its life cycle. A recent study found population structuring of M. vaccinii-corymbosi over a broad spatial scale in the United States. In this study, we examined fine-scale genetic structuring, temporal dynamics, and reproductive biology within a 125-by-132-m blueberry plot from 2010 to 2012. In total, 395 isolates of M. vaccinii-corymbosi were sampled from infected shoots and fruit to examine their multilocus haplotype (MLH) using microsatellite markers. The MLH of 190 single-ascospore isolates from 21 apothecia was also determined. Little to no genetic differentiation and

unrestricted gene flow were detected among four sampled time points and between infected tissue types. Discriminant analysis of principal components suggested genetic structuring within the field, with at least $\mathrm{K}=3$ genetically distinct clusters maintained over four sampled time points. Single-ascospore progeny from eight apothecia had identical MLH and at least two distinct MLH were detected from 13 apothecia. Tests for linkage disequilibrium suggested that genetically diverse ascospore progeny were the product of recombination. This study supports the idea that the fine-scale dynamics of M. vaccinii-corymbosi may be complex, with genetic structuring, inbreeding, and outcrossing detected in the study area.
\end{abstract}

The fungus Monilinia vaccinii-corymbosi (J. M. Reade) Honey causes mummy berry disease of blueberry (Vaccinium spp.) that is characterized by shoot blight followed by fruit infection and mummification (Batra 1983). The life history of the fungus involves overwintering inside desiccated fruit (pseudosclerotia) that lay dormant on the soil surface. In early spring, apothecia are formed from pseudosclerotia and produce wind-disseminated ascospores that infect newly emerging blueberry shoots and leaves and cause blighting (Batra 1983; Cox and Scherm 2001). Conidia produced on blighted shoots are spread primarily by insects to blueberry stigmata, where they exhibit aggressive mimicry to invade the ovary (Batra 1983; Batra and Batra 1985; Cox and Scherm 2001; Ngugi and Scherm 2004). This results in fruit infection and can lead to substantial yield loss in commercial blueberry fields (Batra 1983).

M. vaccinii-corymbosi must undergo both asexual and sexual spore production within a single growing season to complete its life cycle. Research suggests that $M$. vaccinii-corymbosi cannot survive systemically in blueberry shoots, driving the need for ascospore production for shoot infection (Batra 1983). Furthermore, ascospores are not capable of infecting blueberry ovaries, rendering conidia production necessary for the completion of the life cycle (Batra 1983). Both spore types and their infection events on blueberry leaves, shoots, flowers, and fruit contribute to the disease ecology and population dynamics of $M$. vaccinii-corymbosi. It is not currently known whether allele frequencies of $M$. vacciniicorymbosi sampled from infected shoots and fruit are similar within and across several growing seasons. Conidia are the product of asexual reproduction and, in the absence of mutation, they represent an assemblage of genetically identical propagules produced on infected shoots that are capable of infecting blueberry flowers.

Corresponding author: K. M. Burchhardt;

E-mail address: kathleen.burchhardt@gmail.com

*The $\boldsymbol{e}$-Xtra logo stands for "electronic extra" and indicates that two supplementary figures are published online.

(C) 2017 The American Phytopathological Society
Therefore, if conidia that formed on a single infected shoot are spread to numerous flowers, this would result in clonal propagation of the fungus and possibly influence allele frequencies within the population. Because ascospores are the product of sexual reproduction, it is conceivable that ascospore production contributes to genetic diversity within populations of $M$. vacciniicorymbosi. However, the mating system of M. vaccinii-corymbosi is unknown, and other related fungi such as Sclerotinia spp. commonly form ascospores through either outcrossing or selffertilization (Amselem et al. 2011; Heitman et al. 2007; Stewart et al. 2015). Previous research suggested the presence of outcrossing and random mating in several populations of $M$. vaccinii-corymbosi in the northeastern United States, based on high genetic diversity and linkage equilibrium of microsatellite markers (Burchhardt and Cubeta 2015). However, the possibility of inbreeding or selffertilization was suggested in several populations based on the repeated detection of isolates of $M$. vaccinii-corymbosi with the same multilocus haplotype (MLH) and at least one pair of microsatellite markers in significant linkage disequilibrium. Therefore, the population and reproductive biology of M. vacciniicorymbosi needs to be further examined by analyzing allele frequencies and genetic diversity using a large sample size of isolates of M. vaccinii-corymbosi from both infected shoots and fruit within a population sampled over several years from a smaller spatial scale.

An important evolutionary force that contributes to the genetic diversity and structure of field populations of fungi is gene flow (the movement of genetic material). In the case of plant-pathogenic fungi, gene flow can lead to the movement and introgression of alleles that contribute to disease ecology. Therefore, disease management strategies that restrict gene flow are attractive and preferred (McDonald and Linde 2002). Isolates of M. vaccinii-corymbosi collected from 18 fields in the United States were recently analyzed to examine large-scale gene flow and genetic structuring of populations of M. vaccinii-corymbosi (Burchhardt and Cubeta 2015). Strong genetic differentiation and structuring were detected between populations of $M$. vaccinii-corymbosi from blueberry fields in the northeastern United States, suggesting restricted gene flow. Genetic differentiation between isolates of $M$. vaccinii-corymbosi from fields 
spatially separated by as little as $11 \mathrm{~km}$ located in southeastern North Carolina further supported locally restricted gene flow. However, the geographic distance at which gene flow becomes locally restricted has not been examined.

Spatial autocorrelation analysis can be used to determine the geographic scale at which gene flow is restricted by testing for spatial clustering of genetically similar individuals (Peakall and Smouse 2006, 2012). Localized gene flow has been studied within populations of several fungal species using spatial autocorrelation analysis. Brewer et al. (2012) found that gene flow was restricted in populations of the grape (Vitis spp.) pathogen Erysiphe necator at up to $42 \mathrm{~m}$, with spatial aggregation of clonal genotypes within two populations. Everhart and Scherm (2015) conducted threedimensional sampling of Monilinia fructicola within peach (Prunus persica) canopies to detect significant positive spatial autocorrelation at distances $<1 \mathrm{~m}$. More studies are needed to contribute to our knowledge of localized gene flow capabilities of plantpathogenic fungi in agricultural systems.

One component of gene flow is effective spore dispersal, because the dissemination of spores followed by successful infection events results in the movement of the organism and genetic material. The dispersal gradients of ascospores and conidia of $M$. vacciniicorymbosi were previously examined by Cox and Scherm (2001). Either pseudosclerotia with apothecia (and ascospores) or potted plants with infected shoots producing conidia were placed in rows of uninfected potted blueberry plants. Their results suggested short dispersal gradients for both ascospores and conidia. They found that $95 \%$ of blighted shoots were located within $30 \mathrm{~m}$ of an ascospore point source and $95 \%$ of infected fruit were located within $20 \mathrm{~m}$ of a conidial point source. However, the potential for long-distance dispersal was suggested by a long tail in their dispersal gradient curves. Short dispersal gradients of both spore types suggested that gene flow may also be locally restricted within blueberry fields.

Gene flow may be restricted due to geographic, reproductive, or ecological barriers, leading to genetic structuring. Genetic structuring can be detected by using population genetics-based approaches to test for the presence of genetically differentiated populations. Typically, genetic structuring is examined among geographically discrete localities and over large spatial scales. However, it is possible for more than one genetically differentiated population to exist in sympatry. This phenomenon can occur when barriers to sexual recombination or random mating exist, with individuals within an area not having the same probability of mating with each other. Reproductive and ecological barriers can lead to the maintenance of genetically differentiated populations over time. The detection of more than one population in sympatry can also be due to the recent geographic integration and merging of two populations. However, if two or more populations are interfertile and undergo mating, sexual recombination should lead to their genetic admixture and affect allele frequencies over several generations. Temporal sampling within an area can be used to determine whether allele frequencies change over time and to test hypotheses for the presence and maintenance of genetic structure.

Fungi in the phylum Ascomycota typically produce ascospores by either outcrossing or self-fertilization (Heitman et al. 2007; Stewart et al. 2015). The presence of several genetically different isolates of $M$. vaccinii-corymbosi within a fruit may promote outcrossing and generation of genetic diversity via sexual recombination. A mating event between genetically different individuals should result in ascospore progeny that are genetically recombinant. Because $M$. vacciniicorymbosi is haploid, the ascospores also should have a single allele at each locus derived from either the maternal or paternal fungal strain. Neutral genetic markers such as microsatellite markers can be used to determine whether ascospores are genetically recombinant.

In this study, a comprehensive spatial and temporal sampling was conducted to examine the fine-scale population genetics and reproductive biology of $M$. vaccinii-corymbosi. The objectives of this study were to (i) compare allele frequencies of isolates of M. vaccinii-corymbosi collected from infected shoots and fruit within a single growing season; (ii) examine fine-scale genetic structuring using spatial autocorrelation, Bayesian, and multivariate analyses; (iii) examine temporal dynamics and genetic structuring within the field by analyzing isolates of $M$. vacciniicorymbosi collected over three consecutive years; and (iv) analyze genetic diversity in single-ascospore isolates (progeny) sampled from individual apothecia. This study was conducted in a commercial blueberry field in Ivanhoe, NC that was previously determined to contain a population of $M$. vaccinii-corymbosi with high genetic diversity and genetically differentiated from other populations of M. vaccinii-corymbosi at distances as small as $11 \mathrm{~km}$ (Burchhardt and Cubeta 2015). We hypothesized that isolates of M. vaccinii-corymbosi collected over 3 years and from different types of infected plant tissue (i.e., infected shoots and fruit) would not be significantly genetically differentiated and that nonrandom mating would be detected. Because previous research suggested that genetic structuring may be present within the field (Burchhardt and Cubeta 2015), the hypothesis that genetically differentiated populations of $M$. vaccinii-corymbosi exist sympatrically was tested. We hypothesized that there would be evidence for sexual recombination based on high genetic diversity, previously reported in this field (Burchhardt and Cubeta 2015).

\section{MATERIALS AND METHODS}

Sampling and isolation of $M$. vaccinii-corymbosi from infected shoots, fruit, and apothecia. Infected shoots and fruit were sampled from a 50-year-old commercial blueberry farm located in Ivanhoe, NC with a history of mummy berry disease. Samples were collected from a 125-by-132-m plot planted with southern highbush blueberry ( $V$. corymbosum $\times V$. darrowii) in 2002. The plot consisted of 23 rows of 'Legacy' blueberry planted adjacent to 22 rows of 'Blue Ridge' established in 2002. Rows were spaced $3 \mathrm{~m}$ apart in the plot, with approximately 125 blueberry bushes planted at $1-\mathrm{m}$ intervals in each row. Infected shoots with conidia or infected fruit were systematically collected and mapped in 2010, 2011, and 2012. A map of the location where each sample was collected in the plot is provided as Supplementary Figure S1. The systematic sampling strategy was modified in each of the 3 years, with an increase in sampling intensity and coverage over the 3 years. Infected shoots were collected on 6 April 2010 from five rows in each cultivar. Samples were collected from up to six bushes in each row at an interval of at least $3 \mathrm{~m}$ between sampled bushes. In 2011 , infected fruit were collected on 7 June from Blue Ridge and 16 June from Legacy from four rows in each cultivar. A maximum of 16 samples was collected within each row and sampled bushes were spaced at an interval of approximately $5 \mathrm{~m}$ between sampled bushes. In 2012, increased sampling of infected shoots on 3 April and infected fruit on 15 May was performed. On both sampling dates, infected plant material was collected from 10 rows for each cultivar, with samples collected from approximately eight bushes per row spaced at an interval of approximately $15 \mathrm{~m}$ between sampled bushes.

Pure cultures of $M$. vaccinii-corymbosi were obtained via singleconidium isolations from infected shoots or by culturing isolates from inside infected fruit. Single-conidium isolations were performed by scraping conidia off of an infected shoot onto petri dishes containing half-strength potato dextrose agar (PDA) amended to contain $2 \%$ agar (12 $\mathrm{g}$ of Difco potato dextrose broth [PDB] and $20 \mathrm{~g}$ of Difco agar per liter) ([Becton, Dickinson and Company). After incubation at room temperature (approximately $20^{\circ} \mathrm{C}$ ) for at least $12 \mathrm{~h}$, individual germinated conidia were transferred to half-strength PDA containing 1.5\% agar (Difco agar at $15 \mathrm{~g} /$ liter) using a sterile scalpel under a dissecting microscope. Fungal isolations from infected fruit were performed by surface disinfesting fruit for $30 \mathrm{~s}$ with $10 \% \mathrm{NaOCl}, 30 \mathrm{~s}$ with $70 \% \mathrm{EtOH}$, and rinsing in sterile $\mathrm{H}_{2} \mathrm{O}$ for $30 \mathrm{~s}$. Infected fruit were cut in half with a sterile scalpel and a piece of 
infected fruit tissue was removed from a single locule and transferred to half-strength PDA containing $1.5 \%$ agar.

Pseudosclerotia with apothecial initials were arbitrarily collected underneath bushes of Blue Ridge within the plot on 3 March 2011 and 21 February 2013. Pseudosclerotia were placed in a plastic fruit clamshell and stored at $4{ }^{\circ} \mathrm{C}$. A layer of sand approximately $1 \mathrm{~cm}$ thick was added to rectangular glass containers $(10.0$ by 6.0 by $6.5 \mathrm{~cm})$ and distilled water was added until saturation. Pseudosclerotia were transferred to the containers with forceps and placed on the saturated sand, with the stipes of apothecial initials placed upright. The pseudosclerotia were spaced at least $1 \mathrm{~cm}$ apart, with approximately 12 pseudosclerotia per container. Additional distilled water was added to each pseudosclerotium to moisten its surface, and glass lids were placed on top of the containers. The containers were placed under a $40-\mathrm{W}$ fluorescent light with a photoperiod of $14 \mathrm{~h}$ at room temperature (approximately $20^{\circ} \mathrm{C}$ ) for up to 3 weeks to allow for the development of apothecia.

Mature, fully expanded apothecia were excised from pseudosclerotia and affixed to lids of petri dishes containing half-strength PDA amended to 2\% agar (Difco PDB at $12 \mathrm{~g} /$ liter and Difco agar at $20 \mathrm{~g} /$ liter) (Becton, Dickinson and Company) with Vaseline. Individual apothecia removed from a pseudosclerotium were recorded to examine several apothecia produced from the same pseudosclerotium. The apothecia were attached near the edge of the petri dish lid and positioned to release ascospores toward the agar surface. The lid was rotated $90^{\circ}$ every hour for $3 \mathrm{~h}$ to eject ascospores on the agar surface. After incubating at room temperature for at least $12 \mathrm{~h}$, single germinated ascospores were transferred to half-strength PDA plates containing $1.5 \%$ agar (agar at $15 \mathrm{~g} /$ liter) with a sterile scalpel under a dissecting microscope. Up to 14 germinated ascospores were transferred per apothecium.

Isolates of $M$. vaccinii-corymbosi from conidia, infected fruit, and ascospores were grown at room temperature for approximately 2 weeks and then used to inoculate 250-ml Erlenmeyer flasks containing $50 \mathrm{ml}$ of half-strength PDB (Difco PDB at $12 \mathrm{~g} / \mathrm{liter}$ ). The mycelium of each isolate was harvested after approximately 2 weeks by vacuum filtration. Approximately $15 \mathrm{mg}$ of squeezedried mycelium was used for DNA extraction.

DNA analysis with microsatellite markers. DNA was extracted from each isolate of $M$. vaccinii-corymbosi using the cetyltrimethylammonium bromide and chloroform DNA extraction methods outlined by Kretzer et al. (2000), with modifications and DNA precipitation as described by Molecular Ecology Resources Primer Development Consortium et al. (2012). In instances where a fungal culture from infected fruit was not obtained, DNA was extracted directly from the mycelium located in one locule inside an infected fruit using the same protocol. The polymerase chain reaction (PCR) was performed on each DNA extract derived from conidia and infected fruit using nine polymorphic microsatellite markers: M. vaccinii-corymbosi markers 7, 9, 10, 12, 23, 25, 27, 28, and 30 (Molecular Ecology Resources Primer Development Consortium et al. 2012). Eight microsatellite markers were used to analyze single-ascospore isolates: M. vaccinii-corymbosi markers 7, 9, 10, $12,23,27$, and 28. Primer sets for multiple markers were combined in PCR (i.e., multiplexed): markers 7 and 9 (7-9), 12 and 23 (12-23), and 28 and 30 (28-30) were set up in duplexed PCR and markers 10, 25 , and 27 (10-25-27) in triplexed reactions. To fluorescently label PCR products, a CAG sequence was added to the 5 ' end of one of the primers in each microsatellite marker set and an additional CAG primer with either an NED or 6-FAM fluorescent tag attached to the 5 ' end was added to PCR (Molecular Ecology Resources Primer Development Consortium et al. 2012). Reactions were set up in $0.3-\mathrm{ml}, 96$-well PCR plates in $10-\mu \mathrm{l}$ reaction volumes containing $1 \times$ PCR Master Mix (reaction buffer with Taq DNA polymerase at $0.025 \mathrm{U} / \mu \mathrm{l}, 2 \mathrm{mM} \mathrm{MgCl}_{2}$, and $0.2 \mathrm{mM}$ each dNTP) (Thermo Scientific), $0.05 \mu \mathrm{M}$ each CAG-tailed primer, $0.2 \mu \mathrm{M}$ each untailed primer, either $0.6 \mu \mathrm{M}$ (triplexed reactions) or $0.4 \mu \mathrm{M}$ (duplexed reactions) fluorescently labeled CAG primer, and $1.0 \mu \mathrm{l}$ of diluted
DNA extract. PCR from markers 7-9 and 10-25-27 were fluorescently labeled with 6-FAM and reactions containing 12-23 and 28-30 were labeled with NED. Thermocycler conditions were as previously described (Molecular Ecology Resources Primer Development Consortium et al. 2012). PCR products were then multiplexed by combining product from reactions of markers 7-9 with 12-23, as well as product from 10-25-27 with 28-30. Fragment analysis was performed to determine the PCR product length at each locus, as previously described (Molecular Ecology Resources Primer Development Consortium et al. 2012). PCR and fragment analysis conditions for the single-ascospore isolates were identical to those generated from conidia and infected fruit, except that marker 25 was not included in the PCR with 10 and 27, and conditions for duplexed reactions were followed as described above.

The MLH of each sample was determined based on the length of the PCR product (i.e., allele) present at the microsatellite loci. The number of alleles detected at each locus, number of unique MLH, and unbiased haploid diversity were determined with the software GenAlEx version 6.5 (Peakall and Smouse 2006, 2012). GenAlEx was also used to generate clone-corrected datasets by including only one arbitrarily chosen representative isolate of $M$. vacciniicorymbosi per MLH in the dataset. Clone correction was performed separately for isolates collected from the four sampled times: isolates from infected shoots collected in 2010, infected fruit in 2011, and infected shoots and fruit in 2012.

Analyses for genetic differentiation among cultivars, infected plant tissue types, and years. Due to slight differences in timing of bloom and fruit set between Blue Ridge and Legacy, the hypothesis that allele frequencies were statistically similar when comparing isolates of $M$. vaccinii-corymbosi sampled from both cultivars was tested. This was done to confirm that isolates from the cultivars could be pooled in subsequent analyses. Analysis of molecular variance (AMOVA) was performed to test for significant genetic differentiation between the two cultivars. The analysis was performed separately for isolates of $M$. vaccinii-corymbosi from the four sampled time points: isolates from infected shoots collected in 2010, infected fruit in 2011, and infected shoots and fruit in 2012. The analysis was conducted in GenAlEx with 10,000 data permutations and 10,000 pairwise population permutations to calculate $\Phi_{P T}$ values as a measure of genetic differentiation and $P$ values for significance. The analysis was performed using clone-corrected and noncorrected datasets. This enabled us to examine how repeatedly detected MLH in the dataset affected measures of genetic differentiation. Because the two cultivars were not significantly $(\alpha=$ 0.05 ) different in allele frequencies based on clone- and nonclonecorrected data, the data for isolates from the cultivars were pooled in subsequent analyses.

AMOVA was performed to test the hypothesis that isolates of M. vaccinii-corymbosi collected in different years and from infected shoots and fruit within the same growing season were not significantly genetically differentiated. Genetic differentiation between isolates of $M$. vaccinii-corymbosi from the four sampled time points (i.e., isolates from infected shoots collected in 2010, infected fruit collected in 2011, and infected shoots and fruit collected in 2012) was measured based on $\Phi_{P T}$ values. The analysis was performed both with and without clone-correcting isolates from each time point, as described above.

Linkage disequilibrium and analysis of random mating. The statistic $p_{\text {sex }}$, as calculated with the program MLGsim v. 2.0, was used to determine the probability of an MLH being detected a given number of times within the plot (Stenberg et al. 2003). The MLH of isolates from the four sampled time points (2010, 2011, and infected shoots and fruit in 2012) were analyzed separately. $P$ values were calculated based on 1,000 permutations to determine significance at $P<0.05$. Significant $p_{\text {sex }}$ values suggested that isolates associated with a specific MLH were the product of nonrandom mating.

Tests for significant linkage disequilibrium between the nine microsatellite loci were performed using the online implement of 
Genepop version 4.2, with 10,000 dememorizations, 1,000 batches, and 10,000 iterations per batch (Raymond and Rousset 1995; Rousset 2008). Linkage disequilibrium was calculated for both clone-corrected and nonclone-corrected datasets. The analysis was performed separately for isolates of M. vaccinii-corymbosi collected at the four sampled time points $(2010,2011$, and infected shoots and fruit in 2012). The method of Benjamini and Hochberg (1995) was used to correct the $P$ values for multiple comparisons and test for significant linkage disequilibrium (adjusted $P<0.05$ ).

Spatial autocorrelation analysis. The geographic distance at which gene flow is restricted can be examined by testing for clustering of genetically similar isolates located within a certain distance of each other (i.e., distance class). Spatial autocorrelation analysis as calculated with GenAlEx was performed using nonclonecorrected datasets to test whether individuals within distance classes are more genetically similar or more different than expected by random chance. The analysis calculates correlation coefficients $(r)$ based on the genetic similarity of all pairs of individuals that fall within a particular distance class. Spatial autocorrelation was tested by two methods. The first method uses random permutation to shuffle individuals among the geographic locations and generate a $95 \%$ confidence interval of $r$. The null hypothesis of no spatial autocorrelation is supported when the observed value of $r$ lies inside of the $95 \%$ confidence interval of $r$. The other method uses bootstrap estimates of $r$ to place a confidence interval around the observed estimate of $r$. From all sets of pairwise comparisons within a distance class, a preset number of sets are randomly selected from the pool to generate a $95 \%$ confidence interval. The null hypothesis of no spatial autocorrelation is supported when $r=0$ lies within the bootstrap confidence interval. The analysis was performed separately for isolates sampled from infected fruit in 2011, infected shoots in 2012, and infected fruit in 2012. An additional analysis was performed by combining all isolates from infected shoots and fruit collected in 2012 in the analysis. Genetic and geographic distance matrices were produced based on pairwise differences between isolates with GenAlEx. The analysis was performed multiple times using different size distance classes, with the smallest distance class set between 5 and $20 \mathrm{~m}$. The $95 \%$ confidence intervals of $r$ were calculated based on 1,000 random and bootstrap permutations.

Genetic structuring of $M$. vaccinii-corymbosi. Discriminant analysis of principal components (DAPC) as performed with the $\mathrm{R}$ package adegenet was used to further examine genetic structuring within the field using a multivariate analysis approach (Jombart 2008; Jombart et al. 2010). The analysis aims to display differences between clusters of genetically distinct individuals while minimizing variation within the clusters. The clone-corrected dataset, including isolates from 2010, 2011, and 2012, was analyzed.
The K-means cluster algorithm was performed to group samples into clusters and determine the number of supported clusters based on the Bayesian information criterion (BIC) at each value of $\mathrm{K}$. The value of $\mathrm{K}$ at which the BIC begins to either increase or level off can be used to select K to perform DAPC. Scatterplots and histograms were visualized to examine clustering of the samples and assignment of isolates to the clusters. Results from DAPC were further examined with AMOVA by testing for genetic differentiation between isolates assigned to the $\mathrm{K}$ clusters. Each isolate was assigned to one of $\mathrm{K}$ clusters based on the cluster to which they had the highest membership probability. Genetic differentiation between isolates of M. vaccinii-corymbosi assigned to different clusters was calculated with AMOVA, as described above. To further examine genetic differentiation among the clusters, isolates were randomly assigned to clusters and AMOVA was performed to test for significant genetic differentiation among the randomized clusters.

Analysis of single-ascospore isolates. GenAlEx was used to determine the number of ascospores with unique MLH produced by a single apothecium. Mean unbiased haploid diversity was also calculated with GenAlEx for apothecia with a sample size of $\geq 8$ single-ascospore isolates. Two analyses for linkage disequilibrium were performed to test for sexual recombination. First, tests for significant linkage disequilibrium between pairs of the microsatellite markers were performed using the online implement of Genepop version 4.2, as described above. The index of association $\left(I_{A}\right)$ was also calculated as a measure of linkage disequilibrium with the R package poppr using 10,000 permutations to generate $P$ values and test for significance at $P<0.05$ (Kamvar et al. 2014, 2015). Both analyses were conducted separately for each apothecium that had a sample size of $\geq 8$ single-ascospore isolates and produced ascospores with at least two unique MLH.

\section{RESULTS}

Descriptive statistics and analyses for genetic differentiation with AMOVA. Samples of M. vaccinii-corymbosi were collected from 56 infected shoots in 2010, 70 infected fruit in 2011, and 132 infected shoots and 137 infected fruit in 2012 (Table 1). Due to our inability to obtain a pure culture, DNA was directly extracted from mycelium in a single locule within 54 infected fruit in 2011 and 25 infected fruit in 2012. The nine microsatellite loci were polymorphic, ranging from four to nine alleles detected at each locus. High haploid diversity was detected over the four sampled time points (i.e., isolates sampled from infected shoots in 2010, infected fruit in 2011, and infected shoots and fruit in 2012) with a mean of 0.536 (standard error of the mean $[\mathrm{SE}] \pm 0.033$ ). The majority of samples had unique MLH, with 45 MLH detected in

TABLE 1. Descriptive statistics based on analysis of isolates of Monilinia vaccinii-corymbosi collected over 3 years with nine microsatellite markers

\begin{tabular}{|c|c|c|c|c|c|c|c|c|}
\hline Year & Sampled tissue ${ }^{a}$ & Cultivar & $N^{\mathrm{b}}$ & $\Phi_{P T} \mathrm{CC}^{\mathrm{c}}$ & $\Phi_{P T} \mathrm{NC}^{\mathrm{c}}$ & $\mathrm{MLH}^{\mathrm{d}}$ & $h(\mathrm{SE})^{\mathrm{e}}$ & $L^{f}$ \\
\hline \multirow[t]{2}{*}{2010} & Shoots & Blue Ridge & $27(22)$ & $0.006(0.295)$ & $0.007(0.259)$ & 45 & $0.551(0.070)$ & $15 / 3$ \\
\hline & Shoots & Legacy & $29(25)$ & $\ldots$ & $\ldots$ & $\ldots$ & $\ldots$ & $\ldots$ \\
\hline \multirow[t]{2}{*}{2011} & Fruit & Blue Ridge & $26(22)$ & $0(0.630)$ & $0.019(0.080)$ & 56 & $0.507(0.079)$ & $14 / 2$ \\
\hline & Fruit & Legacy & $44(37)$ & $\ldots$ & $\ldots$ & $\ldots$ & $\ldots$ & $\ldots$ \\
\hline \multirow[t]{2}{*}{2012} & Shoots & Blue Ridge & $70(65)$ & $0(0.446)$ & $0.009(0.065)$ & 113 & $0.531(0.065)$ & $6 / 0$ \\
\hline & Shoots & Legacy & $62(55)$ & $\ldots$ & $\ldots$ & $\ldots$ & $\ldots$ & $\ldots$ \\
\hline \multirow[t]{2}{*}{2012} & Fruit & Blue Ridge & $66(65)$ & $0.002(0.337)$ & $0.004(0.208)$ & 121 & $0.555(0.064)$ & $16 / 7$ \\
\hline & Fruit & Legacy & $71(61)$ & $\ldots$ & $\ldots$ & $\ldots$ & $\ldots$ & $\ldots$ \\
\hline
\end{tabular}

a Infected shoots or infected fruit.

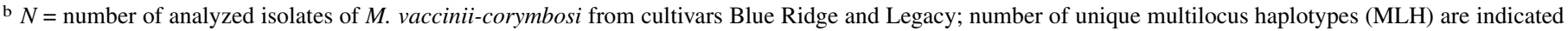
within parentheses.

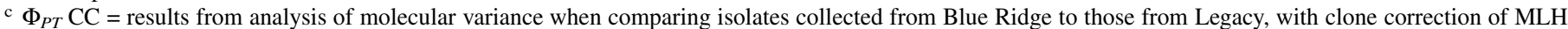
before performing the analysis and $P$ values in parentheses; $\Phi_{P T} \mathrm{NC}=$ results from analysis of molecular variance without clone-correcting isolates collected from Blue Ridge to those from Legacy, with $P$ values in parentheses.

d Number of multilocus haplotypes detected.

e Mean unbiased haploid diversity; SE = standard error of the mean.

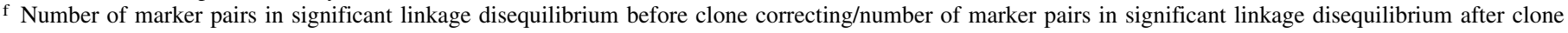
correcting. 
2010, 56 MLH detected in 2011, and 113 MLH detected from infected shoots and 121 from infected fruit in 2012. Isolates with repeatedly detected MLH were identified at the four sampled time points; $20 \mathrm{MLH}$ were detected at least once at two of the four time points, seven MLH at three of the four time points, and two MLH at all four time points.

Isolates of M. vaccinii-corymbosi sampled from Blue Ridge and Legacy were not significantly genetically differentiated based on AMOVA (Table 1). Therefore, data from both cultivars were pooled in subsequent analyses. Analysis of allele frequencies with AMOVA indicated that isolates of $M$. vaccinii-corymbosi from infected shoots and fruit sampled in 2012 were not significantly genetically differentiated when analyzing clone-corrected $\left(\Phi_{P T}=0.005, P=\right.$ $0.072)$ and nonclone-corrected $\left(\Phi_{P T}=0.002, P=0.163\right)$ datasets. When allele frequencies of isolates of $M$. vaccinii-corymbosi from infected shoots sampled in 2010, infected fruit in 2011, and infected shoots and fruit in 2012 were compared, little to no genetic differentiation was detected based on AMOVA (Table 2). Significant genetic differentiation was observed for the comparison of isolates from infected shoots in 2010 with isolates from infected shoots in 2012 when analyzing the nonclone-corrected datasets $\left(\Phi_{P T}=0.013\right.$, $P=0.017)$. However, the comparison was not significant when analyzing clone-corrected datasets $\left(\Phi_{P T}=0.009, P=0.071\right)$.

Nonrandom mating detected based on $p_{\text {sex }}$ and linkage disequilibrium. When analyses were performed separately for isolates of M. vaccinii-corymbosi sampled in 2010 and 2011 and infected shoots and fruit in 2012, two MLH had significant $p_{\text {sex }}$ values in 2010, three MLH in 2011, and one MLH from infected shoots and five MLH from fruit in 2012. The two MLH in 2010 (MLH1 and MLH2) had highly significant $p_{\text {sex }}$ values $(P<1 \mathrm{e}-5)$, with MLH1 represented by four isolates and MLH2 by six isolates. MLH1 and MLH2 were detected multiple times within the other three time points. Seven isolates with MLH1 were detected in 2011, and four isolates were sampled from infected shoots and nine isolates from infected fruit in 2012. The $p_{\text {sex }}$ values for MLH1 were highly significant $(P<1 \mathrm{e}-5)$, with the exception of isolates of M. vaccinii-corymbosi with MLH1 recovered from infected shoots in $2012(P=0.146)$. MLH2 was detected four times in 2011 and six times from infected shoots and twice from infected fruit in 2012. All $p_{\text {sex }}$ values were highly significant $(P<1 \mathrm{e}-5)$, except for isolates from infected fruit in $2012(P=0.159)$. One MLH that was represented by two isolates in 2011 was also significant $(P=0.026)$. The four additional MLH that had significant $p_{\text {sex }}$ values for isolates from fruit in 2012 were represented by two $(P=0.032)$, two $(P=$ $1.23 \mathrm{e}-6)$, two $(P=1 \mathrm{e}-8)$, and three $(P=2.8 \mathrm{e}-6)$ isolates, respectively.

Based on analysis of nonclone-corrected datasets, significant linkage disequilibrium was detected between 15 pairs of microsatellite markers in 2010 (pairs 9-10, 9-12, 9-23, 10-23, 12-23, 12-27, 23-27, 12-28, 23-28, 7-30, 9-30, 10-30, 12-30, 23-30, and 27-30), 14 marker pairs in 2011 (pairs 7-9, 9-12, 10-12, 9-23, 12-23, 12-27, 23-27, 12-28, 27-28, 10-30, 12-30, 23-30, 27-30, and 28-30), six pairs for infected shoots in 2012 (pairs 9-12, 12-23, 12-27, 23-27, 12-28, and 12-30), and 16 pairs for infected fruit in 2012 (pairs 7-9, 9-12, 10-12, 7-23, 9-23, 12-23, 7-27, 9-27, 12-27, 23-27, 9-30, 10-30, 12-30, 23-30, 27-30, and 27-28). After the datasets

TABLE 2. Results from analysis of molecular variance based on isolates of Monilinia vaccinii-corymbosi sampled from infected shoots in 2010, infected fruit in 2011, and from infected shoots and fruit in 2012 (2012 S and $2012 \mathrm{~F}$, respectively) ${ }^{\mathrm{a}}$

\begin{tabular}{lcccc}
\hline$\Phi_{P T}$ & 2010 & 2011 & $2012 \mathrm{~S}$ & $2012 \mathrm{~F}$ \\
\hline 2010 & $\ldots$ & $0.003(0.290)$ & $0.013(0.017)$ & $0.004(0.184)$ \\
2011 & $0.000(0.425)$ & $\ldots$ & $0.006(0.077)$ & $0.001(0.352)$ \\
$2012 \mathrm{~S}$ & $0.009(0.071)$ & $0.009(0.050)$ & $\ldots$ & $0.002(0.163)$ \\
$2012 \mathrm{~F}$ & $0.000(0.436)$ & $0.000(0.436)$ & $0.005(0.072)$ & $\ldots$ \\
\hline
\end{tabular}

a Pairwise $\Phi_{P T}$ values based on analyzing clone-corrected datasets are reported below the diagonal and $\Phi_{P T}$ values using nonclone-corrected datasets above the diagonal, with $P$ values in parentheses. were clone-corrected, three marker pairs were in significant linkage disequilibrium in 2010 (12-23, 23-27, and 23-30), two in 2011 (12-23, 27-30), and none for infected shoots and seven for infected fruit in 2012 (9-12, 7-27, 12-27, 23-27, 27-28, 9-30, and 10-30). None of the marker pairs were consistently in linkage disequilibrium among the four sampling time points, suggesting that linkage disequilibrium was not likely due to physical linkage of the loci.

Lack of positive spatial autocorrelation suggests unrestricted gene flow. Spatial autocorrelation analysis was performed to test for spatial clustering of genetically similar isolates of $M$. vacciniicorymbosi. Separate analyses of isolates of M. vaccinii-corymbosi sampled from infected fruit in 2011, infected shoots in 2012, infected fruit in 2012, and both infected shoots and fruit in 2012 were performed. We did not analyze samples collected in 2010 due to low sample size and unevenly spaced sampling. Regardless of distance class sizes specified in the analyses, no positive spatial autocorrelation was detected. Examples of correlograms from spatial autocorrelation analysis are provided in Supplementary Figure S2.

Presence of genetic structuring within the plot. DAPC suggested that at least three genetically distinct clusters were present within the plot. The exact number of clusters could not be reliably determined because the BIC value continued to decline from $\mathrm{K}=1$ to 10 (Fig. 1). Therefore, results from several independent runs of $\mathrm{K}$-means clustering and DAPC at each value of $\mathrm{K}$ were individually examined to detect the minimum amount of clustering present. Membership assignment of isolates to clusters at $\mathrm{K}>3$ was inconsistent when separate runs of K-means clustering were performed. However, when multiple runs of $K$-means were performed at $K=3$, the isolates were consistently assigned to the same clusters. Clustering was most visually apparent at $\mathrm{K}=3$ (Fig. 2). When the isolates were separated into populations using their cluster assignment with DAPC (Fig. 3), the three clusters were highly significantly genetically differentiated from each other based on AMOVA $\left(\Phi_{P T} \geq 0.202, P \leq\right.$ $0.0001)$. No significant genetic differentiation was detected when isolates were randomly assigned to one of three clusters and AMOVA was performed $\left(\Phi_{P T} \leq 0.001, P \geq 0.353\right)$.

Single-ascospore isolates suggest self-fertilization and outcrossing. In total, 190 single-ascospore isolates were obtained from 21 apothecia sampled from 16 pseudosclerotia. A minimum of 2 and maximum of 14 single-ascospore isolates were generated from each apothecium $($ mean $=9.05, \mathrm{SE}=0.874)$. When analyzing single-ascospore isolates from each apothecium separately, isolates recovered from 8 apothecia had a single MLH per apothecium, while 13 apothecia produced ascospores with more than one genetically unique MLH (Fig. 4). One or two alleles were detected at each microsatellite locus when analyzing isolates from the same apothecium. More than one apothecium was analyzed from four pseudosclerotia. One pseudosclerotium produced three apothecia that were used to generate 36 single-ascospore isolates, all of which

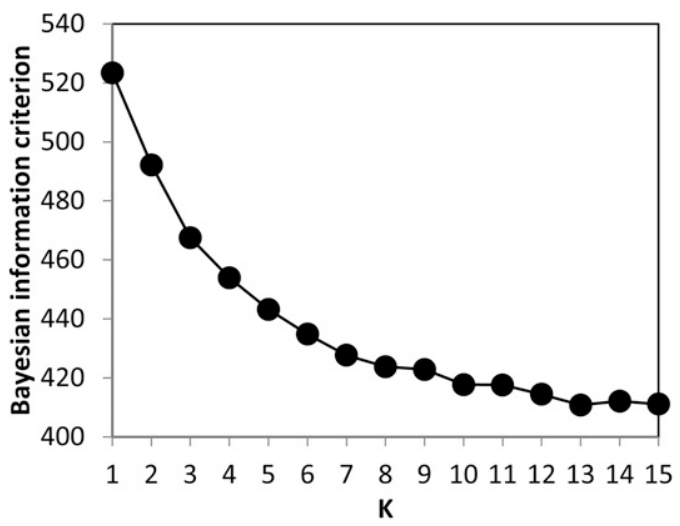

Fig. 1. Bayesian information criterion as calculated with the K-means algorithm implemented in adegenet at each value of $\mathrm{K}$ from 1 to 15 . 
had the same MLH. Another pseudosclerotium produced two apothecia that were used to generate 17 single-ascospore isolates. Sixteen unique MLH were detected from the 17 isolates, with two or three alleles detected at each of the eight microsatellite loci. Examination of 26 single-ascospore isolates sampled from two apothecia on another individual pseudosclerotium identified 13 MLH, with 1 of the MLH represented by isolates sampled from both apothecia. Similarly, nine MLH were detected from 15 examined isolates of M. vaccinii-corymbosi collected from two individual apothecia produced from a single pseudosclerotium collected in 2011, with two of the MLH being detected in both sampled apothecia. Furthermore, one of the MLH detected in both apothecia was also represented by an isolate from an apothecium sampled in 2013. At least eight single-ascospore isolates were obtained from each of 13 apothecia, aiding the reliability of statistical analyses. Tests for linkage disequilibrium could not be conducted on 7 of the 13 apothecia because they produced single-ascospore isolates that had the same MLH. $I_{A}$ was not significant for the six apothecia with at least two MLH detected $(\alpha=0.05)$ (Table 3). No microsatellite marker pairs were in significant linkage disequilibrium (adjusted $\alpha=0.05$ ). The mean haploid diversity for isolates from the six apothecia ranged from 0.187 to 0.536 , with an overall mean of $0.336(\mathrm{SE}=0.046)$.

\section{DISCUSSION}

The most intriguing result of this study was the detection of several genetically differentiated populations of $M$. vacciniicorymbosi within a 125-by-132-m blueberry plot, with maintenance of genetic differentiation over three consecutive years. At least $\mathrm{K}=$ 3 genetically distinct populations were detected within the plot by DAPC. Significant genetic differentiation between isolates of $M$. vaccinii-corymbosi assigned to different genetic clusters with DAPC as determined by AMOVA provided additional support for the presence of multiple genetically distinct populations. Furthermore, when isolates were randomly assigned to clusters and AMOVA was performed, no significant genetic differentiation was detected. The 70 isolates of M. vaccinii-corymbosi sampled from shoots of Blue Ridge in 2012 were included in a previous study on the large-scale population genetics of M. vaccinii-corymbosi (Burchhardt and Cubeta 2015), with DAPC suggesting the existence of at least two genetically distinct populations within the plot. Further genetic structuring was detected in this study by analyzing 395 isolates of M. vaccinii-corymbosi collected from infected shoots and fruit within the plot in 2010, 2011, and 2012.

Temporal maintenance of genetic structure was suggested because isolates with high membership probabilities to the three genetically differentiated populations were detected in all 3 years. Furthermore, AMOVA indicated little to no genetic differentiation among the 3 years despite differences in the sampling scheme used in each year. This suggested that allele frequencies were similar over the 3 years and barriers to gene flow were maintained among the populations. However, significant genetic differentiation was detected between isolates sampled from infected shoots in 2010 and 2012 when analyzing nonclone-corrected datasets. Therefore, the hypothesis that genetic differentiation among samples of $M$. vacciniicorymbosi collected in different years would not be detected was

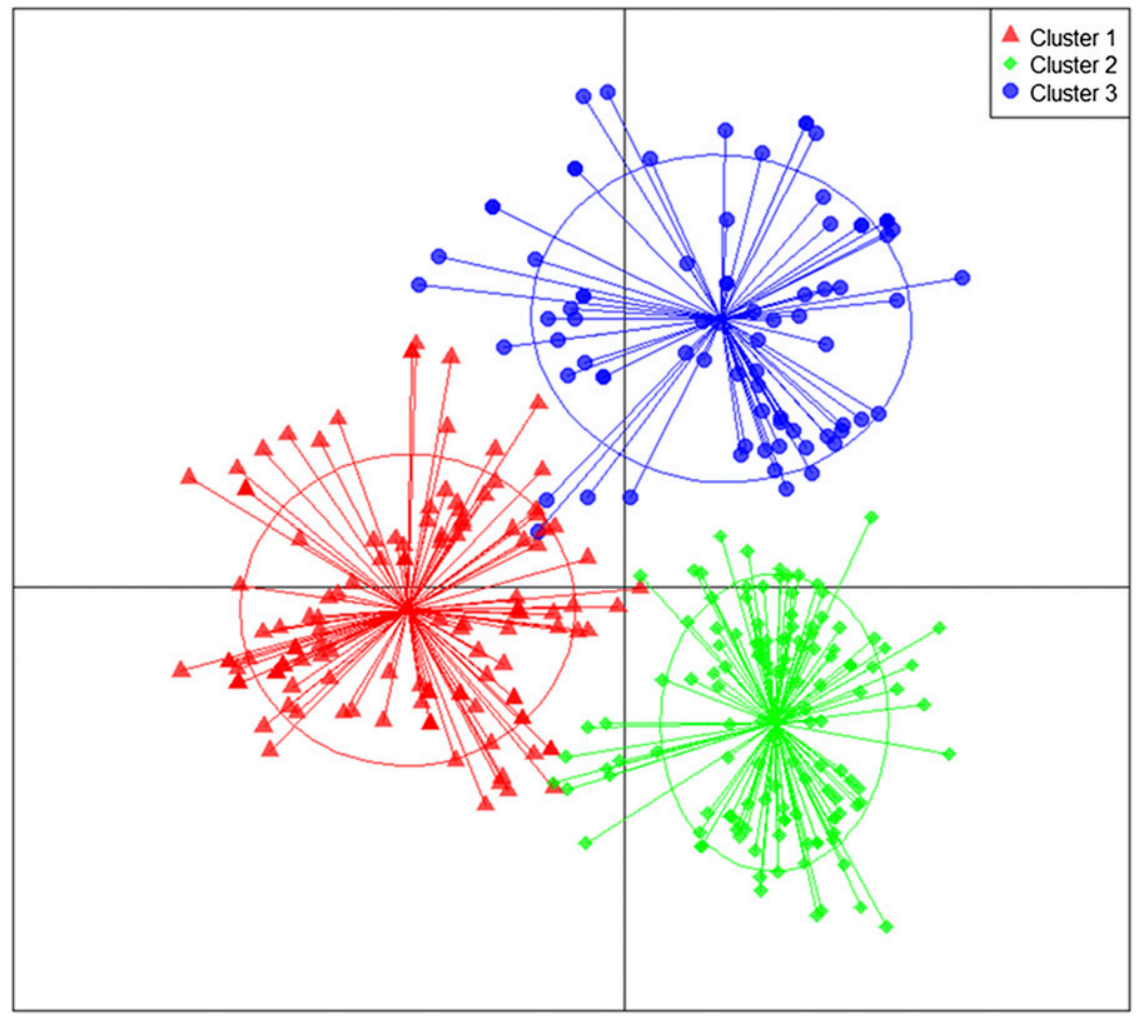

Fig. 2. Scatterplot from discriminant analysis of principal components based on $K=3$ clusters.

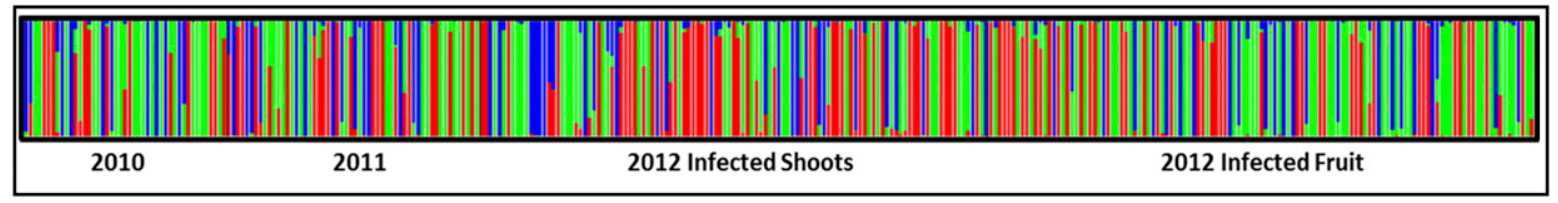

Fig. 3. Membership probabilities of each isolate to $\mathrm{K}=3$ clusters based on discriminant analysis of principal components. 
rejected. The lack of significant genetic differentiation once datasets were clone-corrected demonstrated the impact of clonality on measures of genetic differentiation.

Barriers to gene flow may partially explain the presence and temporal maintenance of several genetically differentiated populations of $M$. vaccinii-corymbosi within the plot. An ecological force that may be driving the maintenance of sympatric genetic differentiation is competition. Conidia of different haplotypes may be more or less aggressive during stigma penetration and ovary infection, with particular haplotypes outcompeting others during ovary colonization. If an individual of $M$. vaccinii-corymbosi outcompetes all other individuals and is the sole haplotype within an infected fruit and is capable of self-fertilization, then selection would favor the maintenance of the haplotype. Recently, isolates of $M$. vaccinii-corymbosi from up to five locules within a single infected fruit were cultured (K. M. Burchhardt, unpublished). Results suggested that genetically different isolates of $M$. vacciniicorymbosi can exist within a single infected fruit, with some harboring more than one isolate with different haplotypes while others contained only a single haplotype. Variation in aggressiveness of individuals has been described in several species of plantpathogenic fungi such as Fusarium graminearum (Chakraborty et al. 2006) and Botrytis cinerea (Reino et al. 2004). Flower inoculation and ovule infection experiments are needed to study aggressiveness and competition of different MLH of $M$. vacciniicorymbosi.

Variation in timing of ascospore and conidia production also may enforce sympatric genetic differentiation. Research from previous investigations suggests that pseudosclerotia can vary in timing of apothecia production on different species of blueberry and cultivars within species (Lehman and Oudemans 1997, 2000; Scherm et al. 2001). The research plot in this study was neighbored by plots of other blueberry cultivars on the commercial farm, with the possibility of spore dispersal and gene flow among plots. Furthermore, apothecia production from pseudosclerotia collected underneath bushes of a single blueberry cultivar can occur over several weeks (Lehman and Oudemans 1997), and a single apothecium may produce ascospores over several days (Lehman and Oudemans 2000). It is possible that individuals of $M$. vaccinii-corymbosi with different haplotypes produce apothecia and ascospores at different times, resulting in differences in timing of shoot infection. Variation in the incubation and latent periods following ascospore infection and production of conidia has not been studied. If differences in timing of ascospore production results in differences in timing of conidia production, then

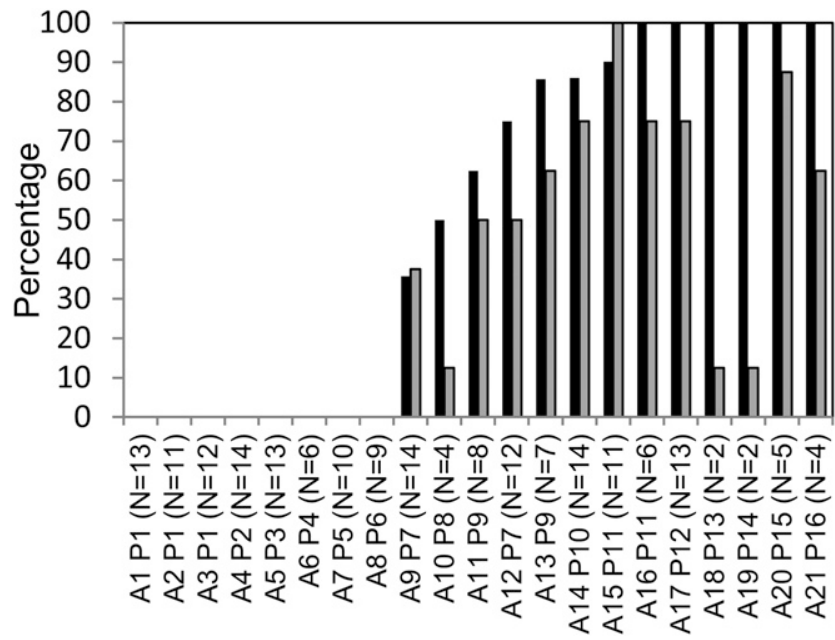

Fig. 4. Analysis of single-ascospore isolates of Monilinia vaccinii-corymbosi from 21 apothecia. $\mathrm{A}=$ apothecium number, $\mathrm{P}=$ pseudosclerotium number that the apothecium was derived from, and $\mathrm{N}=$ number of single-ascospore isolates analyzed. Black bars indicate percent genetically unique single-ascospore isolates and gray bars denote percentage of polymorphic loci. haplotypes that produce conidia within the same time frame should be more likely to coinoculate a flower, allowing them to undergo sexual recombination. Timing of conidia production is particularly critical because flowers are most receptive to infection on the day of anthesis, with decreasing susceptibility over time (Ngugi et al. 2002). Batra (1983) reported that conidia produced from a single infected shoot are only viable for up to 6 days and conidia production can occur within a field over a period of 1 month. Further research to examine how the reproductive biology and ecology of $M$. vaccinii-corymbosi influence its genetic structure is warranted.

Because infected shoots and fruit are the result of infection by sexual (ascospores) and asexual (conidia) propagules, respectively, the hypothesis that allele frequencies will be similar when analyzing isolates of $M$. vaccinii-corymbosi sampled from infected shoots and fruit within a single growing season was tested. AMOVA suggested that isolates from infected shoots and fruit were not genetically differentiated regardless of the presence of repeatedly detected MLH. Therefore, the hypothesis that allele frequencies are similar between isolates of $M$. vaccinii-corymbosi collected from infected shoots and fruit within the same growing season was accepted. These results are consistent with those of Everhart and Scherm (2015), who found that isolates of M. fructicola recovered from blighted peach blossoms and infected fruit were not significantly genetically differentiated. Another recent study by Stewart et al. (2015) did not detect genetic differentiation between isolates of Exobasidium maculosum from infected blueberry leaves and fruit.

Nonrandom association of alleles at unlinked, neutral loci can be caused by deviation from random mating and detected through analysis of linkage disequilibrium between loci. Although haplotypic diversity was high each year, nonrandom mating was suggested based on the presence of linkage disequilibrium and repeatedly recovered MLH over 3 years of sampling that were statistically unlikely to be the product of random mating. Interestingly, two MLH (MLH1 and MLH2) were detected numerous times within each year of sampling, had highly significant $p_{\text {sex }}$ values, and were always assigned to different clusters with DAPC, suggesting their genetic dissimilarity (data not shown). Therefore, these results suggest clonal propagation of these two MLH.

No restricted gene flow was detected within the examined 125by-132-m plot. This was unexpected because previous research suggested that asexual and sexual spore dispersal occurs primarily within $30 \mathrm{~m}$ of a source (Cox and Scherm 2001). There are several plausible explanations for the absence of positive spatial autocorrelation within the plot. First, although the sampling area was chosen based on previous research on spore dispersal capabilities (Cox and Scherm 2001), the sampling area may not have been large

TABLE 3. Descriptive statistics for 13 apothecia with single-ascospore isolate sample sizes greater than eight ${ }^{\mathrm{a}}$

\begin{tabular}{lrccc}
\hline Sample ID & $N$ & MLH & $I_{A}$ & $u h$ \\
\hline A1 P1 & 13 & 1 & $\ldots$ & $\ldots$ \\
A2 P1 & 11 & 1 & $\ldots$ & $\ldots$ \\
A3 P1 & 12 & 1 & $\ldots$ & $\ldots$ \\
A4 P2 & 14 & 1 & $\ldots$ & $\ldots$ \\
A5 P3 & 13 & 1 & $\ldots$ & $\ldots$ \\
A7 P5 & 10 & 1 & $\ldots$ & $\ldots$ \\
A8 P6 & 9 & 1 & $0.2503(0.0772)$ & $0.187(0.092)$ \\
A9 P7 & 14 & 5 & $-0.0592(0.667)$ & $0.272(0.103)$ \\
A11 P9 & 8 & 5 & $0.0623(0.3604)$ & $0.267(0.101)$ \\
A12 P7 & 12 & 9 & $0.0446(0.3673)$ & $0.378(0.083)$ \\
A14 P10 & 14 & 12 & $0.2565(0.0986)$ & $0.536(0.006)$ \\
A15 P11 & 11 & 10 & $-0.2967(0.9991)$ & $0.378(0.083)$ \\
A17 P12 & 13 & 13 & $\ldots$
\end{tabular}

a Sample ID lists the apothecium (A) and pseudosclerotium (P) number, $N=$ number of single-ascospore isolates, $\mathrm{MLH}=$ number of unique multilocus haplotypes detected, $I_{A}=$ index of association (with $P$ value in parentheses), and $u h=$ haploid diversity (with standard error in parentheses); ... indicates that the analysis was not performed due to an MLH sample size of one. 
enough to detect aggregation of haplotypes. To test this hypothesis, the sampling area would need to be enlarged to a scale at which restricted gene flow could be detected. It is also possible that the presence of several genetically differentiated populations of M. vaccinii-corymbosi within the plot influenced the results from spatial autocorrelation analysis. This may have occurred if genetically similar isolates that belonged to the same population were in a distance class that also contained isolates that were strongly genetically divergent and belonged to a different population. Genetic structuring caused by factors other than geography needs to be examined further. Finally, the sampling scheme within the plot may not have been sufficient for detecting restricted gene flow in M. vaccinii-corymbosi. Although samples of infected shoots and fruit were systematically collected, the sampling scheme did not account for the possible presence of hierarchical structuring. At each sampled locality within the plot, M. vaccinii-corymbosi was isolated from a single infected shoot or fruit. Genetic diversity of isolates of $M$. vaccinii-corymbosi in the canopy of a single blueberry bush should be examined. Everhart and Scherm (2015) reported high genetic diversity within the canopy of a single peach tree. The study was also the first to use three-dimensional mapping to detect positive spatial autocorrelation at $<1 \mathrm{~m}$ in canopies, demonstrating the importance of hierarchical sampling. Furthermore, $M$. vaccinii-corymbosi was arbitrarily isolated from a single locule of an infected fruit to obtain pure cultures or extract DNA. It was recently determined that several genetically unique isolates of $M$. vaccinii-corymbosi can be isolated from different locules in a single infected fruit (K. M. Burchhardt, unpublished). Genetic diversity of isolates of $M$. vaccinii-corymbosi recovered from a single fruit and from an individual bush should be examined further.

Our results indicate that apothecia can produce sexually recombinant ascospores with unique MLH, while others may produce ascospores with identical MLH. Single-ascospore isolates have been studied in other fungi to examine sexual recombination. Gladieux et al. (2011) used microsatellite markers to determine that pseudothecia of Venturia inaequalis can produce genetically different ascospore progeny. In contrast, the fungus Teratosphaeria nubilosa produced pseudothecia with genetically identical ascospore progeny based on analysis with microsatellite markers (Pérez et al. 2010). A mixed mating system with both self-fertilization and outcrossing was suggested in several populations of the chestnut blight fungus Cryphonectria parasitica by analyzing ascospores from perithecia (Marra et al. 2004; Milgroom et al. 1993). This study further demonstrates that singleascospore isolates are useful for elucidating and inferring the reproductive biology of fungi.

Some fungi are known to have mixed mating systems that involve both outcrossing and self-fertilization. For example, the mating system of Sclerotinia trifoliorum has been classified as "bipolar heterothallic" and allows for self-fertilization and outcrossing (Ekins et al. 2006; Uhm and Fujii 1983a,b). The eight ascospores within an ascus of $S$. trifoliorum are dimorphic, with four ascospores being smaller than the other four. The larger ascospores are selffertile, while the smaller ascospores are self-sterile and can only be fertilized by spermatia (microconidia) of larger ascospore isolates (Uhm and Fujii 1983a, 1983b). Interestingly, Batra (1983) previously reported three types of ascospores produced by $M$. vacciniicorymbosi based on their morphology. The first ascospore type produces granules and one or two germ tubes, and has a normal cytoplasm. The second ascospore type does not have granules, has an alveolate cytoplasm, and may produce abortive germ tubes and microconidia. The third type of ascospore is smaller, does not have granules, has a scant cytoplasm, and does not produce germ tubes or microconidia. Research is needed to determine whether ascospores with different morphologies influence mating processes in $M$. vacciniicorymbosi.

Outcrossing was detected because 13 apothecia produced singleascospore isolates with at least two different MLH. Because polymorphisms were commonly detected at more than one locus, it is unlikely that genetic differences were due to mutation alone. Furthermore, analyses for linkage disequilibrium suggested that the ascospores were the result of sexual recombination. Eight apothecia were inferred to be the product of self-fertilization because they produced single-ascospore isolates with the same MLH. However, mating between closely related isolates could also result in ascospore isolates with identical MLH. This explanation seems unlikely based on high genetic diversity and polymorphisms at the microsatellite markers examined. Further support for self-fertilization in M. vaccinii-corymbosi was the detection of an MLH (MLH1) within four infected fruit that was previously detected multiple times over 3 years of sampling. Our results suggested that this repeatedly detected MLH was highly unlikely to be the product of random mating. Additional experiments are needed to better confirm that isolates with repeatedly detected MLH are capable of self-fertilizing.

Results from this study suggest that the population and reproductive biology of $M$. vaccinii-corymbosi are complex and warrant further examination. In this study, it was determined that genetic structuring existed within a 125-by-132-m plot and genetic structure was maintained over 3 years. Linkage disequilibrium and repeatedly detected MLH with significant $p_{\text {sex }}$ values supported nonrandom mating within the plot. Spatial autocorrelation analysis supported lack of aggregation of haplotypes. Apothecia may produce genetically identical or sexual recombinant ascospores. It is important to consider the reproductive biology of plant-pathogenic fungi to develop appropriate disease management strategies (McDonald and Linde 2002). Sexual recombination can aid in creating progeny capable of surviving in the presence of management strategies such as fungicides and genes for resistance. Selfing via homothallic reproduction and asexual reproduction can propagate and maintain isolates with advantageous combinations of alleles. Thus, fungi with mixed mating systems such as $M$. vaccinii-corymbosi have been hypothesized to possess a greater evolutionary advantage and, therefore, are more challenging to manage (McDonald and Linde 2002). Research is needed to further elucidate the mating system and examine hierarchical structuring of populations of $M$. vacciniicorymbosi.

\section{ACKNOWLEDGMENTS}

We thank M. McCormick, B. Bloodworth, D. Gentry, and S. Rose for their help collecting samples; and A. Ballard for aiding in culturing M. vaccinii-corymbosi from infected tissues. Funding for this project was provided by a North Carolina Department of Agriculture \& Consumer Sciences Specialty Crops Block Grant to M. Cubeta and a P.E.O. Scholar Award from the International Chapter of the P.E.O. Sisterhood to K. M. Burchhardt.

\section{LITERATURE CITED}

Amselem, J., Cuomo, C. A., van Kan, J. A. L., Viaud, M., Benito, E. P., Couloux, A., Coutinho, P. M., de Vries, R. P., Dyer, P. S., Fillinger, S., Fournier, E., Gout, L., Hahn, M., Kohn, L., Lapalu, N., Plummer, K. M., Pradier, J.-M., Quévillon, E., Sharon, A., Simon, A., ten Have, A., Tudzynski, B., Tudzynski, P., Wincker, P., Andrew, M., Anthouard, V., Beever, R. E., Beffa, R., Benoit, I., Bouzid, O., Brault, B., Chen, Z., Choquer, M., Collémare, J., Cotton, P., Danchin, E. G., Da Silva, C., Gautier, A., Giraud, C., Giraud, T., Gonzalez, C., Grossetete, S., Güldener, U., Henrissat, B., Howlett, B. J., Kodira, C., Kretschmer, M., Lappartient, A., Leroch, M., Levis, C., Mauceli, E., Neuvéglise, C., Oeser, B., Pearson, M., Poulain, J., Poussereau, N., Quesneville, H., Rascle, C., Schumacher, J., Ségurens, B., Sexton, A., Silva, E., Sirven, C., Soanes, D. M., Talbot, N. J., Templeton, M., Yandava, C., Yarden, O., Zeng, Q., Rollins, J. A., Lebrun, M.-H., and Dickman, M. 2011. Genomic analysis of the necrotrophic fungal pathogens Sclerotinia sclerotiorum and Botrytis cinerea. PLoS Genet. 7:e1002230.

Batra, L. R. 1983. Monilinia vaccinii-corymbosi: Its biology on blueberry and comparison with related species. Mycologia 75:131-152.

Batra, L. R., and Batra, S. W. T. 1985. Floral mimicry induced by mummyberry fungus exploits hosts pollinators as vectors. Science 228:10111013. 
Benjamini, Y., and Hochberg, Y. 1995. Controlling the false discovery rate- a practical and powerful approach to multiple testing. J. R. Stat. Soc. B 57:289-300.

Brewer, M. T., Frenkel, O., and Milgroom, M. G. 2012. Linkage disequilibrium and spatial aggregation of genotypes in sexually reproducing populations of Erysiphe necator. Phytopathology 102:997-1005.

Burchhardt, K. M., and Cubeta, M. A. 2015. Population structure of the blueberry pathogen Monilinia vaccinii-corymbosi in the United States. Phytopathology 105:533-41.

Chakraborty, S., Liu, C. J., Mitter, V., Scott, J. B., Akinsanmi, O. A., Ali, S., Dill-Macky, R., Nicol, J., Backhouse, D., and Simpfendorfer, S. 2006. Pathogen population structure and epidemiology are keys to wheat crown rot and Fusarium head blight management. Australas. Plant Pathol. 35:643-655.

Cox, K. D., and Scherm, H. 2001. Gradients of primary and secondary infection by Monilinia vaccinii-corymbosi from point sources of ascospores and conidia. Plant Dis. 85:955-959.

Ekins, M., Aitken, E. A., and Coulter, K. C. 2006. Homothallism in Sclerotinia minor. Mycol. Res. 110:1193-1199.

Everhart, S. E., and Scherm, H. 2015. Fine-scale genetic structure of Monilinia fructicola during brown rot epidemics within individual peach tree canopies. Phytopathology 105:542-9.

Gladieux, P., Guerin, F., Giraud, T., Caffier, V., Lemaire, C., Parisi, L., Didelot, F., and Le Cam, B. 2011. Emergence of novel fungal pathogens by ecological speciation: Importance of the reduced viability of immigrants. Mol. Ecol. 20:4521-4532.

Heitman, J., Kronstad, J. W., Taylor, J. W., and Casselton, L. A. 2007. Sex in Fungi: Molecular Determination and Evolutionary Implications. ASM Press, Washington, D.C.

Jombart, T. 2008. adegenet: A R package for the multivariate analysis of genetic markers. Bioinformatics 24:1403-1405.

Jombart, T., Devillard, S., and Balloux, F. 2010. Discriminant analysis of principal components: A new method for the analysis of genetically structured populations. BMC Genet. 11:94.

Kamvar, Z. N., Tabima, J. F., Grünwald, N. J. 2014. Poppr: An R package for genetic analysis of populations with clonal, partially clonal, and/or sexual reproduction. PeerJ 2:e281.

Kamvar, Z. N., Brooks, J. C., and Grünwald, N. J. 2015. Novel R tools for analysis of genome-wide population genetic data with emphasis on clonality. Front. Genet. 6:208

Kretzer, A. M., Molina, R., and Spatafora, J. W. 2000. Microsatellite markers for the ectomycorrhizal basidiomycete Rhizopogon vinicolor. Mol. Ecol. 9:1190-1191.

Lehman, J. S., and Oudemans, P. V. 1997. Phenology of apothecium production in populations of Monilinia vaccinii-corymbosi from early- and late-maturing blueberry cultivars. Phytopathology 87:218-223.

Lehman, J. S., and Oudemans, P. V. 2000. Variation and heritability of phenology in the fungus Monilinia vaccinii-corymbosi on blueberry. Phytopathology 90:390-395.

Marra, R. E., Cortesi, P., Bissegger, M., and Milgroom, M. G. 2004. Mixed mating in natural populations of the chestnut blight fungus, Cryphonectria parasitica. Heredity 93:189-195.

McDonald, B. A., and Linde, C. 2002. The population genetics of plant pathogens and breeding strategies for durable resistance. Euphytica 124:163-180.
Milgroom, M. G., Lipari, S. E., Ennos, R. A., and Liu, Y.-C. 1993. Estimation of the outcrossing rate in the chestnut blight fungus, Cryphonectria parasitica. Heredity 70:385-392.

Molecular Ecology Resources Primer Development Consortium, Abelló, P., Ai, W., Altmann, C., Bernardi, G., Bonato, O., Burchhardt, K. M., Chen, X., Chen, Z., Č́žzová, D., Clouet, C., Cubeta, M. A., Garcia-Merchan, V. H., Gauthier, N., Gibson, S., Halačka, K., Hamdi, F., Hankeln, T., Hochkirch, A., Hrbek, T., Jackson, A. M., Lin, C., Lin, S.-M., MacPherson, E., Macrander, J., Marešová, E. V. A., Mendel, J. A. N., Nowak, M., Orti, G., Palero, F., Papoušek, I. V. O., Pascual, M., Schmitt, T., Semmens, B. X., Streito, J.-C., Tian, E.-W., Tseng, S.-P., Veith, M., Vetešník, L., Wang, H.-Y., Weyer, J., Willis, S., Yu, H. U. I., and Zhou, Z. 2012. Permanent genetic resources added to molecular ecology resources database 1 April 2012-31 May 2012. Mol. Ecol. Resour. 12:972-974.

Ngugi, H. K., and Scherm, H. 2004. Pollen mimicry during infection of blueberry flowers by conidia of Monilinia vaccinii-corymbosi. Physiol. Mol. Plant Pathol. 64:113-123.

Ngugi, H. K., Scherm, H., and Lehman, J. S. 2002. Relationships between blueberry flower age, pollination, and conidial infection by Monilinia vaccinii-corymbosi. Phytopathology 92:1104-1109.

Peakall, R., and Smouse, P. E. 2006. GENALEX 6: Genetic analysis in Excel. Population genetic software for teaching and research. Mol. Ecol. Notes 6: 288-295.

Peakall, R., and Smouse, P. E. 2012. GenAlEx 6.5: Genetic analysis in Excel. Population genetic software for teaching and research-An update. Bioinformatics 28:2537-2539.

Pérez, G., Slippers, B., Wingfield, B. D., Hunter, G. C., and Wingfield, M. J. 2010. Micro- and macrospatial scale analyses illustrates mixed mating strategies and extensive geneflow in populations of an invasive haploid pathogen. Mol. Ecol. 19:1801-1813.

Raymond, M., and Rousset, F. 1995. GENEPOP (version 1.2): Population genetics software for exact tests and ecumenicism. J. Hered. 86:248-249.

Reino, J. L., Hernández-Galán, R., Durán-Patrón, R., and Collado, I. G. 2004. Virulence-toxin production relationship in isolates of the plant pathogenic fungus Botrytis cinerea. J. Phytopathol. 152:563-566.

Rousset, F. 2008. Genepop'007: A complete reimplementation of the Genepop software for Windows and Linux. Mol. Ecol. Resour. 8:103-106.

Scherm, H., Savelle, A. T., and Pusey, P. L. 2001. Interactions between chillhours and degree-days affect carpogenic germination in Monilinia vacciniicorymbosi. Phytopathology 91:77-83.

Stenberg, P., Lundmark, M., and Saura, A. 2003. MLGsim: A program for detecting clones using a simulation approach. Mol. Ecol. Notes 3: 329-331.

Stewart, J. E., Brooks, K., Brannen, P. M., Cline, W. O., and Brewer, M. T. 2015. Elevated genetic diversity in the emerging blueberry pathogen Exobasidium maculosum. PLoS One 10. doi:10.1371/journal.pone.0132545

Uhm, J. Y., and Fujii, H. 1983a. Ascospore dimorphism in Sclerotinia trifoliorum and cultural characters of strains from different-sized spores. Phytopathology 73:565-569.

Uhm, J. Y., and Fujii, H. 1983b. Heterothallism and mating type mutation in Sclerotinia trifoliorum. Phytopathology 73:569-572. 